\title{
A Randomized Double-blind Study on the Efficacy of $20 \%$ VCO Cream versus a Commercial Emollient for Atopic Dermatitis in Children
}

\author{
Gina C. Castro ${ }^{1,2}$ and Jovencio G. Apostol ${ }^{1,2,3}$ \\ ${ }^{1}$ The Graduate School, University of Santo Tomas, España Blvd., Sampaloc, Manila, Philippines \\ ${ }^{2}$ Faculty of Pharmacy, University of Santo Tomas, España Blvd., Sampaloc, Manila, Philippines \\ ${ }^{3}$ Research Center for the Natural and Applied Sciences, University of Santo Tomas, España Blvd., Sampaloc, Manila, Philippines
}

\begin{abstract}
Objective. To assess the effects of a formulated $20 \%$ VCO cream on symptoms of atopic dermatitis in children relative to a commercial emollient with skin barrier protective property indicated for dry, itchy skin.

Methods. In a randomized, double-blind, pilot study, pediatric patients with atopic dermatitis according to the modified Hanifin and Rajka criteria were enrolled and assigned to use either formulated VCO cream or commercial emollient. Treatments were applied twice daily for four (4) weeks. Outcome measures were investigator- and patientassessed clinical efficacy based on Severity Scoring of Atopic Dermatitis (SCORAD) severity index, and incidences of documented adverse events.

Results. Twenty-nine patients were recruited in the study and in an intention-to-treat analysis, mean SCORAD indices were reduced by $41.79 \%$ and $29.77 \%$ in the VCO cream group and commercial emollient group, respectively. Both study groups showed significant reduction in the mean subjective SCORAD index relating to pruritus and sleep loss. Mean objective SCORAD index, based on intensity items and total surface with eczema, was also significantly improved in the VCO cream group after four weeks of product usage. The study products were generally well-tolerated, with minor adverse events reported for the VCO cream group.
\end{abstract}

Conclusion. Results of the study suggest that application of VCO at $20 \%$ in a cream formulation is more effective than the tested commercial emollient in alleviating symptoms of $A D$ in children.

Key Words: atopic dermatitis, children, pilot study, virgin coconut oil

\section{INTRODUCTION}

Paper presented at the UST Faculty of Pharmacy Research Forum, March 12, 2019, Main Bldg., University of Santo Tomas, España Blvd., Sampaloc, Manila, Philippines.

Corresponding author: Gina C. Castro, RPh, PhD Faculty of Pharmacy University of Santo Tomas

España Blvd., Sampaloc, Manila 1008, Philippines Email: gnacastro2006@gmail.com
Atopic dermatitis $(\mathrm{AD})$ is a common chronic disorder characterized by xerosis, eczematous lesions, and pruritus. It affects mostly children and is known to adversely influence the quality of life (QoL) of patients. ${ }^{1}$ Topical corticosteroids are the gold standard of anti-inflammatory therapy in AD. However, use of corticosteroids is associated with cutaneous effects like skin atrophy, purpura, striae, telangiectasia, focal hypertrichosis, and acneiform or rosacea-like eruptions. The application of potent corticosteroids on large areas of the body for prolonged periods may even lead to systemic effects like pituitary-adrenal axis suppression and retardation of growth in children. ${ }^{2}$ Recent developments in the understanding of the pathophysiology of the epidermal barrier has led to the exploration of alternative, non-steroid therapies that control pruritus, suppress inflammation and/or repair skin barriers. ${ }^{3}$ The regular use of emollients is prescribed for active 
disease flares, in combination with topical anti-inflammatory agents, and as maintenance therapy in $\mathrm{AD} .^{4-6}$

Virgin coconut oil (VCO) is a naturally-derived ingredient known to possess moisturizing and antiinflammatory properties. ${ }^{7}$ In a clinical trial among patients with mild to moderate xerosis, $\mathrm{VCO}$ was shown to be as safe and effective as mineral oil in alleviating symptoms of skin dryness. ${ }^{8}$ A moisturizing lotion incorporated with VCOloaded solid lipid particles (VCO-SLPs) increased skin moisture and enhanced skin elasticity in healthy subjects. ${ }^{9}$ Preliminary studies have shown that topical application of pure VCO improved SCORAD (SCORing Atopic Dermatitis - a clinical tool for assessing the severity of atopic dermatitis) severity index values, transepidermal water loss, and skin capacitance in pediatric $\mathrm{AD}$ patients. ${ }^{10}$ It also significantly reduced objective SCORAD severity index and Staphylococcus aureus colonization in adult $\mathrm{AD}$ patients. ${ }^{11}$

A systematic review identified the Severity Scoring of Atopic Dermatitis (SCORAD) index as one of the most suitable instruments for disease severity evaluation in $\mathrm{AD} .{ }^{12}$ The SCORAD index employs an assessment tool to determine (a) the extent of the disease, (b) the intensity according to erythema, edema/papules, oozing/crust, excoriation, lichenification, and dryness, and (c) subjective symptoms based on pruritus and sleep loss. ${ }^{13}$

The use of VCO as main active ingredient in an emollient product for $\mathrm{AD}$ has not yet been reported. Formulation of VCO into a suitable dosage form is important to make administration easier and thereby improve patient compliance. The authors developed a $20 \%$ VCO cream and sought to assess its effects on symptoms of childhood atopic dermatitis relative to a commercial emollient using the SCORAD assessment tool.

\section{MATERIALS AND METHODS}

\section{Study design and setting}

The study protocol was approved by the Graduate School Ethics Review Committee, University of Santo Tomas. This was a randomized, double-blind, pilot clinical trial performed to evaluate the effects of a formulated $20 \%$ VCO cream in comparison to a commercial emollient with skin barrier protective effects indicated for dry, itchy skin in improving $\mathrm{AD}$ symptoms in children, as measured by SCORAD index values. Signed and dated written informed consent were obtained from one parent/guardian for children less than 7 years old, and signed and dated written assent were obtained from subjects aged 7 to 18 with signed endorsement of their parent/guardian. The study was conducted over a two-month period through a communitybased dermatology clinic in Navotas City, Philippines.

\section{Participants}

Pediatric patients (male/female, aged 1-18 years old) with $\mathrm{AD}$ according to the modified Hanifin and Rajka criteria ${ }^{9}$ were enrolled in the study. Patients who were under any medication, or had other skin disorders were excluded.

To detect an effect size of 1.137 in the mean subjective SCORAD scores with two-sided level of significance and $80 \%$ power of the statistical test, a minimum of 9 participants were included in each study group. This effect size was based on the report of Padilla-Evangelista and co-workers, ${ }^{10}$ where mean scores before and after treatment with $\mathrm{VCO}$ were $6.51(\mathrm{SD}=3.08)$ and $2.12(\mathrm{SD}=2.33)$, respectively. $\mathrm{G}^{*}$ Power 3.12 was used in the calculations.

\section{Intervention}

Subjects were randomized using Microsoft Excel program and assigned to receive either formulated 20\% VCO cream (composed of water, VCO, cetyl acohol, hydroxyethyl acrylate/sodium acryloyldimethyltaurate copolymer and squalane and polysorbate 60 , ceteth- 6 , sodium benzoate, and citric acid) or a commercial emollient (composed of water, Olea europeae fruit oil, glycerin, pentylene glycol, Olus oil, Elaeis guineensis oil, hydrogenated lecithin, squalane, betaine, palmitamide MEA, sarcosine, acetamide MEA, hydroxyethylcellulose, carbomer, sodium carbomer, and xanthan gum).

The chosen comparator emollient is a non-steroid firstline treatment commonly prescribed by dermatologists in clinical practice for the management of atopic dermatitis symptoms in the Philippines. Patients and/or the accompanying parent/guardian were interviewed. Clinical evaluation was done by a dermatologist before treatment (baseline), and at weeks 1, 2, and 4 using the standard SCORAD Index Evaluation Form.

Study products were supplied in 50-gram coded white plastic jars and dispensed by study pharmacist. The patient's parents were instructed to apply the assigned cream on affected skin areas twice daily, in the morning and at night. Follow-up visits were conducted to allow dermatologist examination, compliance check, product replenishment, and photograph documentation. Efficacy was evaluated by another dermatologist using objective SCORAD parameters for total surface with eczema, and intensity items (erythema, edema, oozing, excoriation, lichenification, and dryness). Patient assessment was based on subjective SCORAD parameters of pruritus, and sleep loss.

\section{Outcomes}

The primary outcome was to compare the change in mean objective and subjective SCORAD scores between the formulated VCO Cream and the commercial emollient after four weeks of use. The secondary outcome was to determine tolerability of formulated VCO cream based on incidences of documented adverse events (AE).

\section{Statistical analysis}

Mean age of two groups were compared using Student's t-test, while gender association was made with Fisher's exact 
test. Comparison of categorical data between groups utilized Mann-Whitney U-Test. Change in parameter scores from baseline within treatment groups was evaluated by Wilcoxon Signed Rank Test. All analyses were performed with significance level of 0.05 .

\section{RESULTS}

\section{Participant characteristics}

Of the 40 individuals screened, 29 met the entry criteria and were randomized to the treatment $(\mathrm{VCO}, \mathrm{n}=15)$, and control (commercial, $n=14$ ) (Figure 1). Among these, 6 were considered lost to follow-up due to non-appearance in any scheduled visits, while 5 were withdrawn because of protocol violation, adverse events, and consent withdrawal.

Using an intention-to-treat analysis, all 29 patients were included in the full analysis. For all missing values, last observation was carried forward. Baseline scores for defining parameters of the VCO cream and commercial emollient groups were comparable $(p>0.05)$ (Table 1$)$.

\section{SCORAD index values}

Mean overall SCORAD indices were reduced by $41.79 \%$ in the VCO cream group, and by $29.77 \%$ in the commercial emollient group (Table 2). Subjective symptoms of pruritus and sleep loss significantly improved after four weeks of treatment in both groups.

There was also observed reduction in the mean objective SCORAD scores, represented by intensity of eczema and total surface with eczema, but was only significant for the VCO cream group $(p=0.008)$ (Table 3$)$.

Most patients observed that their skin was less dry and itchy, and became softer and smoother with use of the study products. Visible improvement in intensity of eczema was observed in patients who presented with more severe symptoms at the start of the study. Representative photographs of subjects from both study groups before and after treatment are shown in Figure 2.

\section{Adverse events}

The study products were generally well-tolerated. One subject in the VCO cream group reported transient, localized reddening and mild itching on Day 3 of application, which did not recur with continued use of the cream. Two subjects in the VCO cream group dropped-out due to reported localized urticaria after a few days of product use, which resolved spontaneously. No adverse events were reported for the commercial moisturizing cream group.

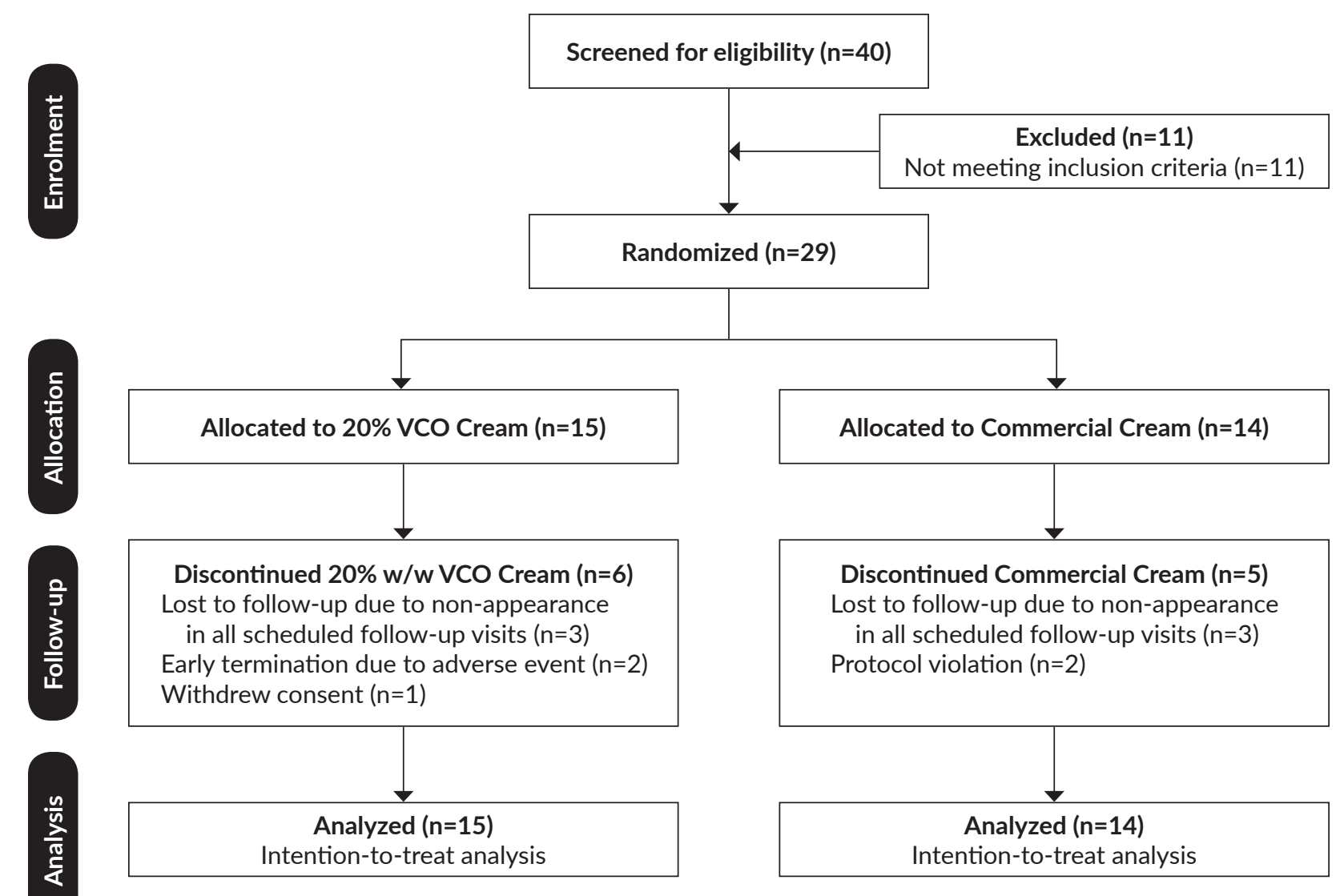

Figure 1. Flow Diagram of Subject Disposition. 
Table 1. Demographic characteristics of the study population $(n=29)$

\begin{tabular}{lccc}
\multicolumn{1}{c}{ Characteristics } & 20\% VCO Cream (n=15) & Commercial Emollient (n=14) & $\boldsymbol{p}$-value \\
Age (years) & $6.40 \pm 5.05$ & $5.29 \pm 4.16$ & $0.524^{\left({ }^{(a)}\right.}$ \\
Gender: Female & $7(46.7 \%)$ & $10(71.4 \%)$ & $1.000^{(\mathrm{b})}$ \\
SCORAD index at baseline, mean \pm SD & & & \\
$\quad$ Overall & $32.95 \pm 16.89$ & $30.37 \pm 17.27$ & $0.727^{(\mathrm{c})}$ \\
Intensity and extent (objective) & $25.69 \pm 13.56$ & $23.44 \pm 13.06$ & $0.662^{(\mathrm{c})}$ \\
$\quad$ Pruritus and sleep loss (subjective) & $7.27 \pm 6.08$ & $6.93 \pm 6.40$ & $0.913^{(\mathrm{c})}$ \\
\hline
\end{tabular}

(a) $p$-values based on Student's t-test

(b) $p$-values based on Fischer's exact test

(c) $p$-values based on Mann-Whitney $U$ test

Table 2. Individual SCORAD scores for clinical trial respondents

A. VCO Cream

\begin{tabular}{|c|c|c|c|c|c|c|}
\hline \multirow{2}{*}{ Patient Code } & \multicolumn{2}{|c|}{ Objective SCORAD } & \multicolumn{2}{|c|}{ Subjective SCORAD } & \multicolumn{2}{|c|}{ Total SCORAD } \\
\hline & Baseline & Post-treatment & Baseline & Post-treatment & Baseline & Post-treatment \\
\hline ADP-03 & 11.5 & 9.3 & 3.0 & 1.0 & 14.5 & 10.3 \\
\hline ADP-05 & 7.6 & 7.6 & 0.0 & 0.0 & 7.6 & 7.6 \\
\hline ADP-10 & 19.1 & 19.1 & 4.0 & 4.0 & 23.1 & 23.1 \\
\hline ADP-11 & 11.7 & 11.7 & 4.0 & 4.0 & 15.7 & 15.7 \\
\hline ADP-12 & 42.6 & 8.5 & 0.0 & 0.0 & 42.6 & 8.5 \\
\hline ADP-16 & 15.8 & 15.8 & 2.0 & 2.0 & 17.8 & 17.8 \\
\hline ADP-17 & 44.2 & 19.7 & 1.0 & 0.0 & 45.2 & 19.7 \\
\hline ADP-18 & 39.6 & 39.4 & 15.0 & 7.0 & 54.6 & 46.4 \\
\hline ADP-19 & 26.4 & 3.5 & 11.0 & 0.0 & 37.4 & 3.5 \\
\hline ADP-21 & 34.3 & 34.3 & 10.0 & 10.0 & 44.3 & 44.3 \\
\hline ADP-22 & 26.1 & 26.1 & 8.0 & 8.0 & 34.1 & 34.1 \\
\hline ADP-23 & 13.3 & 7.4 & 8.0 & 0.0 & 21.3 & 7.4 \\
\hline ADP-24 & 24.7 & 11.2 & 8.0 & 0.0 & 32.7 & 11.2 \\
\hline ADP-27 & 50.2 & 17.9 & 20.0 & 13.0 & 70.2 & 30.9 \\
\hline ADP-28 & 18.2 & 7.2 & 15.0 & 0.0 & 33.2 & 7.2 \\
\hline \multicolumn{5}{|c|}{ Average Total SCORAD Index Values } & 32.95 & 19.18 \\
\hline & & & \multicolumn{2}{|c|}{ Net Change in SCORAD Index Values } & \multicolumn{2}{|c|}{$41.79 \%$} \\
\hline
\end{tabular}

\section{B. Commercial Emollient}

\begin{tabular}{|c|c|c|c|c|c|c|}
\hline \multirow{2}{*}{ Patient Code } & \multicolumn{2}{|c|}{ Objective SCORAD } & \multicolumn{2}{|c|}{ Subjective SCORAD } & \multicolumn{2}{|c|}{ Total SCORAD } \\
\hline & Baseline & Post-treatment & Baseline & Post-treatment & Baseline & Post-treatment \\
\hline ADP-01 & 3.9 & 7.2 & 0.0 & 0.0 & 3.9 & 7.2 \\
\hline ADP-02 & 15.2 & 15.2 & 4.0 & 4.0 & 19.2 & 19.2 \\
\hline ADP-04 & 15.8 & 11.7 & 5.0 & 6.0 & 20.8 & 17.7 \\
\hline ADP-06 & 24.9 & 25.1 & 17.0 & 3.0 & 41.9 & 28.1 \\
\hline ADP-07 & 19.7 & 21.5 & 0.0 & 0.0 & 19.7 & 21.5 \\
\hline ADP-08 & 16.3 & 12.8 & 0.0 & 0.0 & 16.3 & 12.8 \\
\hline ADP-09 & 41.6 & 12.5 & 12.0 & 2.0 & 53.6 & 14.5 \\
\hline ADP-13 & 17.9 & 7.2 & 11.0 & 0.0 & 28.9 & 7.2 \\
\hline ADP-14 & 8.0 & 10.7 & 0.0 & 0.0 & 8.0 & 10.7 \\
\hline ADP-15 & 24.7 & 28.8 & 2.0 & 0.0 & 26.7 & 28.8 \\
\hline ADP-20 & 42.8 & 3.5 & 16.0 & 0.0 & 58.8 & 3.5 \\
\hline ADP-25 & 39.8 & 39.8 & 10.0 & 10.0 & 49.8 & 49.8 \\
\hline ADP-26 & 15.7 & 15.7 & 15.0 & 15.0 & 30.7 & 30.7 \\
\hline \multirow[t]{3}{*}{ ADP-29 } & 41.9 & 41.9 & 5.0 & 5.0 & 46.9 & 46.9 \\
\hline & & & \multicolumn{2}{|c|}{ Average Total SCORAD Index Values } & 30.4 & 21.3 \\
\hline & & & \multicolumn{2}{|c|}{ Net Change in SCORAD Index Values } & \multicolumn{2}{|c|}{$29.77 \%$} \\
\hline
\end{tabular}

Table 3. Mean values for clinical parameters at baseline and post-treatment

\begin{tabular}{|c|c|c|c|c|c|c|}
\hline \multirow{2}{*}{ Measure } & \multicolumn{3}{|c|}{$20 \%$ VCO Cream } & \multicolumn{3}{|c|}{ Commercial Emollient } \\
\hline & Baseline & Post-treatment & $p$-value* & Baseline & Post-treatment & $p$-value* \\
\hline Objective SCORAD & 25.69 & 15.91 & 0.008 & 23.44 & 18.11 & 0.262 \\
\hline Subjective SCORAD & 7.27 & 3.27 & 0.011 & 6.93 & 3.21 & 0.046 \\
\hline
\end{tabular}

${ }^{*} p$-values are based on Wilcoxon Signed Rank test. 

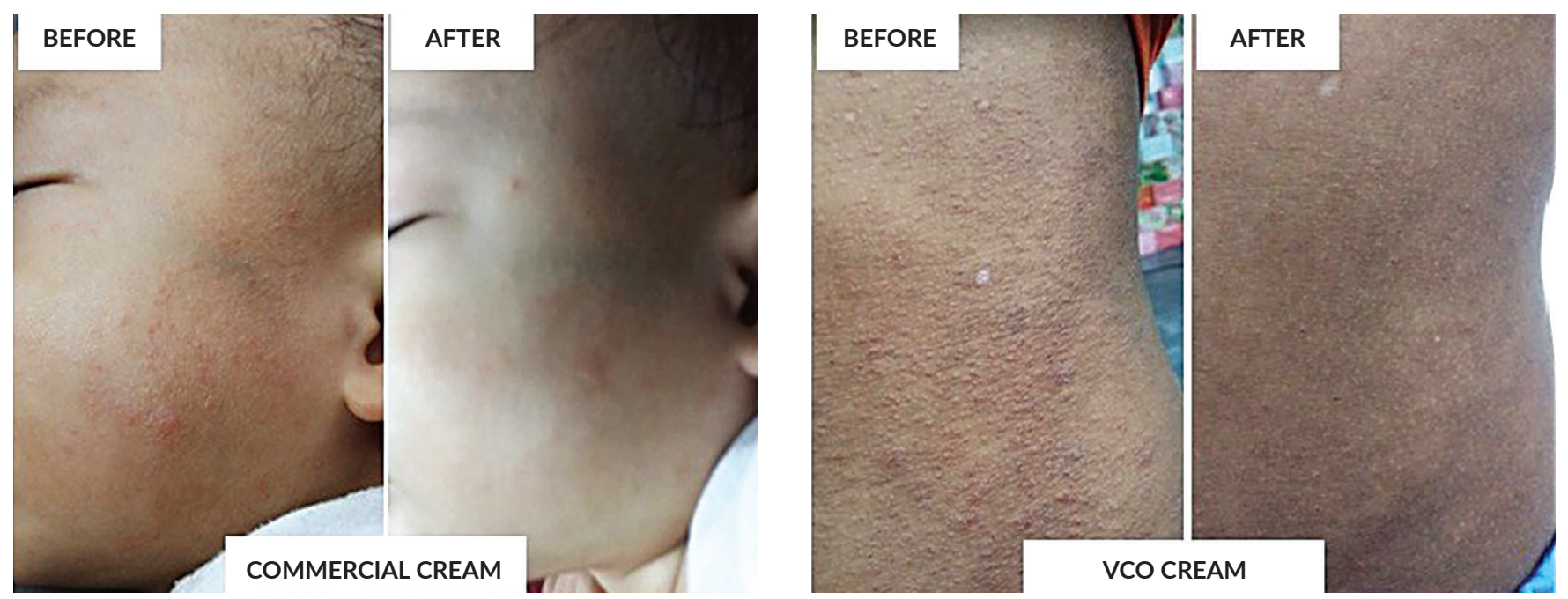

Figure 2. Representative photographs of study subjects before and after 4 weeks of treatment.

\section{DISCUSSION}

The present study revealed that both $20 \%$ VCO cream and commercial emollient significantly improved mean overall SCORAD scores, including mean subjective SCORAD values represented by pruritus and sleep loss, in pediatric $\mathrm{AD}$ patients. This may be attributed to the moisturizing and anti-inflammatory properties of the active ingredients in both creams. Reduction in pruritus and associated sleep loss symptoms are known to positively impact the QoL of AD patients.

Topical application of pure $\mathrm{VCO}$ has been reported to promote wound-healing owing to the presence of minor bioactives and antibacterial fatty acids. ${ }^{14}$ This may partly account for the significant reduction of objective SCORAD scores, represented by intensity and total surface with eczema, seen only in the VCO cream group. Observation by patients who completed the study in both groups also support the beneficial effects of using $\mathrm{VCO}$ cream and the commercial emollient on skin dryness, itching, and texture.

The use of the formulated VCO cream was not associated with any serious adverse events. Definite association with treatment of the two reported cases of urticaria in the VCO cream group cannot be established since both subjects returned for follow-up only after three weeks from occurrence of reported $\mathrm{AE}$, and acute urticaria is known to be of varied etiology. $\mathrm{AE}$ in both cases resolved spontaneously with no medical intervention.

Emollients are the mainstay for both reactive and proactive treatment approaches for $\mathrm{AD} .{ }^{15}$ Results of the study strongly suggest that the formulated 20\% VCO cream could be a suitable non-steroid emollient to help ease associated symptoms of $\mathrm{AD}$ in children.

\section{CONCLUSION}

The study results provide evidence-based support that application of $\mathrm{VCO}$ at $20 \%$ in a cream formulation is more effective than the tested commercial emollient in alleviating symptoms of $\mathrm{AD}$ in children. 20\% VCO cream showed a potential to be more beneficial than tested commercial emollient, although this would require validation in larger controlled trials.

A follow-up clinical trial among larger population in an institutional setting is ideal to validate the preliminary findings in this study.

It is recommended that design of study protocol is considered on minimizing study drop-outs, since ability of pediatric patients to comply with treatment regimen and follow-up visits are largely influenced by the availability of parents/guardians. The potential steroid-sparing effect of the $\mathrm{VCO}$ cream as an adjunct in AD therapy may also be explored.

\section{Statement of Authorship}

Castro prepared the study protocol, performed data collection and analysis, and drafted initial paper. Apostol reviewed protocol design, provided insights and oversight during conduct of clinical trial, and reviewed final paper. All authors approved the final version submitted.

\section{Author Disclosure}

All authors declared no conflicts of interest.

\section{Funding Source}

This paper was funded by the Department of Science and Technology - Science Education Institute (DOSTSEI), Commission on Higher Education (CHED), and University of Santo Tomas (UST) Grants Office. 


\section{REFERENCES}

1. Eichenfield LF, Tom WL, Chamlin SL, Feldman SR, Hanifin JM, Simpson EL, et al. Guidelines of care for the management of atopic dermatitis - Section 1. Diagnosis and assessment of atopic dermatitis. J Am Acad Dermatol. 2014; 70(2) 338-51.

2. Hengge UR, Ruzicka T, Schwartz RA, Cork MJ. Adverse effects of topical glucocorticosteroids. J Am Acad Dermatol. 2006; 54(1): 1-15.

3. Valdman-Grinshpoun Y, Ben-Amitai D, Zvulunov A. Barrierrestoring therapies in atopic dermatitis: Current approaches and future perspectives. Dermatol Res Pract. 2012; 2012:923134.

4. Rubel D, Thirumoorthy T, Soebaryo RW, Weng SC, Gabriel TM, Villafuerte LL, et al. Consensus guidelines for the management of atopic dermatitis: An Asia-Pacific perspective. J Dermatol. 2013; 40(3):160-71.

5. Conde-Taboada A, Gonzales-Barcala FJ, Toribio J. Review and update of current understanding of childhood atopic dermatitis. Actas Dermosifiliogr. 2008; 99(9):690-700.

6. Shekariah T, Kalavala M, Alfaham M. Atopic dermatitis in children: a practical approach. Paediatr Child Health. 2011; 21(3):112-8.

7. Logeswari P, Kumar VD, Usha PTA, Kumar P. In vivo antiinflammatory effect of emu oil (Dromais novaehollandiae) and virgin coconut oil (Cocos nucifera) on phorbol ester induced acute inflammatory model. Int J Pharm Sci Res. 2014; 5(3): 896-99. doi: 10.13040/IJPSR.0975-8232.5(3).896-99.

8. Agero AL, Verallo-Rowell VM. A randomized double-blind controlled trial comparing extra virgin coconut oil with mineral oil as a moisturizer for mild to moderate xerosis. Dermatitis. 2004; 15(3):109-16.
9. Noor NM, Aziz AA, Sarmidi MR, Aziz R. The effect of virgin coconut oil loaded solid lipid particles (VCO-SLPs) on skin hydration and skin elasticity. Jurnal Teknologi 2013; 62(1):39-43.

10. Padilla-Evangelista MT, Abad-Casintahan F, Lopez-Villafuerte $\mathrm{L}$. The effect of topical virgin coconut oil on SCORAD index, transepidermal waree loss, and skin capacitance in mild to moderate pediatric atopic dermatitis: a randomized, double-blind, clinical trial. Int J Dermatol. 2014; 53:100-8.

11. Verallo-Rowell VM, Dillague KM, Syah-Tjundawan BS. Novel antibacterial and emollient effects of coconut and virgin olive oils in adult atopic dermatitis. Dermatitis. 2008; 19(6):308-15.

12. Schmitt J, Langan S, Deckert S, Svensson A, von Kobyletzki L, Thomas K, et al. Assessment of clinical signs of atopic dermatitis: A systematic review and recommendation. J Allergy Clin Immunol. 2013; 132(6):1337-47.

13. Oranje AP, Glazenburg EJ, Wolkerstorfer A, de Waard-van der Spek FB. Practical issues on interpretation of scoring atopic dermatitis: the SCORAD index, Objective SCORAD and the Three-Item Severity Score. Br J Dermatol. 2007; 157(4):645-8.

14. Nevin KG, Rajamohan T. Effect of topical application of virgin coconut oil on skin components and antioxidant status during dermal wound healing in young rats. Skin Pharmacol Physiol. 2010; 23(6):290-7.

15. Wollenberg A, Bieber T. Proactive therapy of atopic dermatitis an emerging concept. Allergy. 2009; 64(2):276-8. 